\begin{tabular}{|c|c|}
\hline $\begin{array}{l}\text { Chemistry of } \\
\text { Metals and Alloys }\end{array}$ & $\begin{array}{l}\text { Chem. Met. Alloys } 4 \text { (2011) 31-37 } \\
\text { Ivan Franko National University of LLiv } \\
\text { www.chemetatlijournalorg }\end{array}$ \\
\hline
\end{tabular}

\title{
Activation of thin layers of two aromatic hydrocarbons
}

\author{
Sylwester KANIA ${ }^{1,2} *$, Janusz KULIŃSKI ${ }^{2}$ \\ ${ }^{1}$ Institute of Physics, Technical University of Lodz, \\ Wólczańska St. 219, 93-009 Łódź, Poland \\ ${ }^{2}$ Centre of Mathematics and Physics, Technical University of Lodz, \\ Al. Politechniki 11, 90-924 Łódź, Poland \\ * Corresponding author. Tel.: +48-42-6313614; fax: +48-42-6313614; e-mail: skania@p.lodz.pl
}

Received June 15, 2010; accepted May 18, 2011; available on-line November 8, 2011

The kinetics of activation currents induced by ethanol into thin layers of two aromatic hydrocarbons were investigated. The results indicate a partial chemisorption process with injection of carriers. Assuming that the increase of the current over the initial value is proportional to the covered surface, the enthalpies determined from the slope of the $\mathrm{C}_{2} \mathrm{H}_{5} \mathrm{OH}$ adsorption dependence were nearly identical for tetracene and for p-quaterphenyl. This suggests a similar mechanism for the effect of adsorption on the electron structure of the adsorbing molecule for both of the substances. Variations of the conductivity were observed due to the adsorption of ethanol, which suggests that hopping through localized states is the dominant mode of charge transport.

\section{Charge transport / Acenes / Adsorption / Tetracene / p-quaterphenyl}

\section{Introduction}

There exist many papers in the scope of physics of organic molecules adsorbed on covalent semiconductor surfaces. Covalent materials have high melting point, and this causes difficulties for applications. Acenes are a group of materials with low melting point, and have the advantage that they are well characterized. Studies intended to applications of organic layers in high-tech industry such as chemical sensors and molecular electronic devices are now in progress [1-4]. A broken surface of covalent silicon possesses a grid of dangling bonds, and the adsorption points are properly defined. An adsorbed ethanol molecule provides the two electrons required for the new covalent bond, and two kinds of sorption are possible: physisorption with activation energy ranging from $0.93 \mathrm{eV}$ to $1.52 \mathrm{eV}$ and chemisorption with two step dissociation with activation energy from $2.46 \mathrm{eV}$ to $4.29 \mathrm{eV}[5,6]$. Changes of the density of states indicate substantial modification of the valence and conduction bands of $\mathrm{Si}$ due to the adsorption of ethanol affecting the electrical properties of the surface. It is under question if there exists a possibility to achieve a similar process for adsorption of ethanol on the surface of acenes, which have no dangling bonds but posses a grid of active points that could make hydrogen bonds between the adsorbing small dicarbon molecule of ethanol and the $\pi$ bond existing between two neighbouring carbons in the ring $[7,8]$. But if this process would occur, then the modulation depth of the conductivity should be wider than for silicon, which has rather high intrinsic conductivity. Acene crystals are built from molecules with a covalent core, but the intermolecular interactions are dispersive with non directional bonding. It has been shown from quantum-mechanical calculations that the cohesive energy in the solid state between two adjacent benzene rings is ca $486 \mathrm{meV} /$ molecule [9] and this is approximately the value that is needed for an ethanol molecule to dissociate between two adjacent parallel rings. But for the planar surface the aromatic rings act as hydrogen bond acceptors for the donor group of ethanol [7,8]. Tetracene and p-quaterphenyl have the same number of carbon rings in the molecule, and very similar crystallization properties such as density of packing and coordination number, but different construction of the molecule. The molecular weight is above $200 \mathrm{~g} / \mathrm{mol}$ and the overall length between 12 and $16 \AA$. The small activator molecule of ethanol, with $\mathrm{ca} 3$ times shorter effective diameter, has a head similar to the water molecule in the form of a C-O unit weakly bonded to an $\mathrm{H}$ atom via the oxygen. The weight of this head is 
Table 1 Increase of the mobility in tetracene and p-quaterphenyl due to $\mathrm{O}_{2}$ and $\mathrm{H}_{2} \mathrm{O}$ adsorption.

\begin{tabular}{l|l|c|c|c}
\hline \multirow{2}{*}{ Compound } & Charge carriers & \multicolumn{2}{|c|}{ In vacuum } & In air \\
\cline { 2 - 4 } & & $\begin{array}{c}\text { Mobility } \\
{\left[\mathrm{cm}^{2} / \mathrm{Vs}\right]}\end{array}$ & $\begin{array}{c}\text { Activation energy } \\
{[\mathrm{eV}]}\end{array}$ & $\begin{array}{c}\text { Mobility } \\
{\left[\mathrm{cm}^{2} / \mathrm{Vs}\right]}\end{array}$ \\
\hline tetracene & electrons & $4 \cdot 10^{-4}$ & 0.03 & $7 \cdot 10^{-4}$ \\
p-quaterphenyl & holes & $2.0 \cdot 10^{-5}$ & 0.04 & $9 \cdot 10^{-5}$ \\
& electrons & $1.0 \cdot 10^{-5}$ & 0.03 & $1.7 \cdot 10^{-4}$ \\
\hline
\end{tabular}

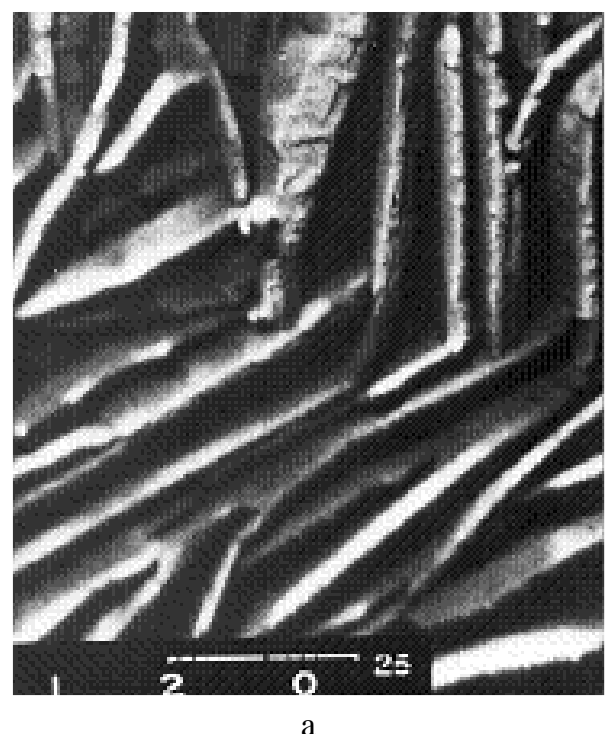

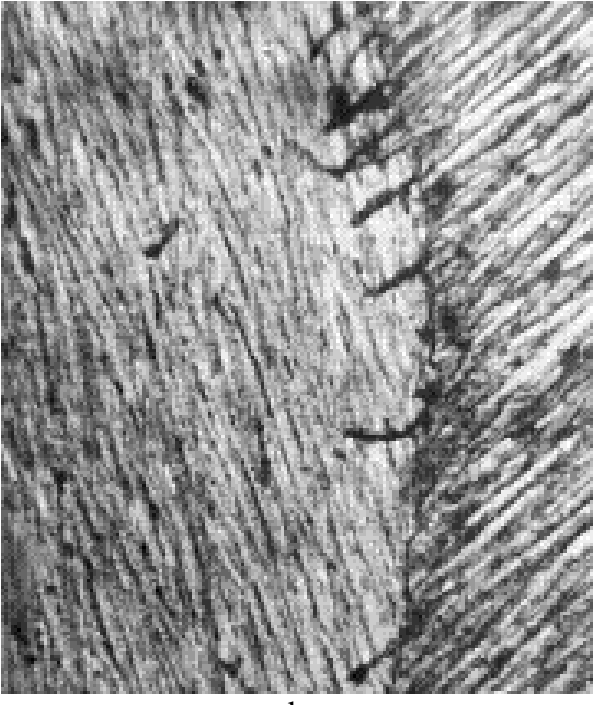

b

Fig. 1 Surface of a polycrystalline p-quaterphenyl film; (a) image obtained with an electron microscope, (b) image obtained with an optical microscope.

two times more than the weight of the non-reactive tail built from $\mathrm{CH}_{3}$, so we assume that the head is decisive for adsorption.

\section{Experimental}

Thin films of the "sandwich" type with $\mathrm{Au}-\mathrm{Al}$ electrodes and polycrystalline tetracene $\left(\mathrm{C}_{18} \mathrm{H}_{12}\right)$ or p-quaterphenyl $\left(\mathrm{C}_{24} \mathrm{H}_{18}\right)$ were prepared by evaporation in vacuum under a pressure of the order of $10^{-5}$ Torr on a glass plate covered by an $\mathrm{Au}$ electrode. The temperature of the substrate was about $300 \mathrm{~K}$ and the evaporation rate was varied within the range 20-30 ̊/s. X-ray diffraction structural examinations of the layers were made using an automatic diffractometer DAR in the $2 \theta$ range from $5^{\circ}$ to $80^{\circ}$ with a step of $0.05^{\circ}$. Microscopic observations revealed grains at the surface with sizes of the order of magnitude of one micrometer (Fig. 1). The thickness of the films was about $4 \mu \mathrm{m}$ for p-quaterphenyl and from about $15 \mu \mathrm{m}$ to $20 \mu \mathrm{m}$ for tetracene. The layers were fixed in a vacuum chamber in a vacuum of approximately $10^{-2}$ Torr and were then subjected to the action of the activator vapour. $\mathrm{C}_{2} \mathrm{H}_{5} \mathrm{OH}$ is characterized by high polarity with a permanent dipole momentum 1.7 D depending on the presence of the
$\mathrm{OH}$ group, which probably makes possible the sorption process.

The time dependence of the current characteristics of the acenes under an increasing concentration of ethanol in the vacuum chamber was investigated. The electrical measurement device in Fig. 2, similar to the one used in $[1,10]$, consists of a current source and an electronic $I-t$ recorder. The initial ethanol pressure was 0.2 Torr and the measurements were performed at room temperature. One recording cycle with multiple changing of the ethanol concentration was for tetracene $1800 \mathrm{~s}$ and for p-quaterphenyl $2400 \mathrm{~s}$ and all the measurements ended after rapid evacuation of the system. The electrical response measured in ethanol vapour showed a decrease of the resistance of the films. The situation was reversed when the gas flow was shut off, with a rapid decrease of the current to a level one order of magnitude above the original one.

\section{Results and discussion}

Earlier works $[1,4]$ have revealed an increase of the conductivity in layers of tetracene and p-quaterphenyl in an external atmosphere of $\mathrm{O}_{2}$ and of steam $\left(\mathrm{H}_{2} \mathrm{O}\right)$ (Table 1). Such a process with one order of magnitude growth of the dark current was interpreted as surface 


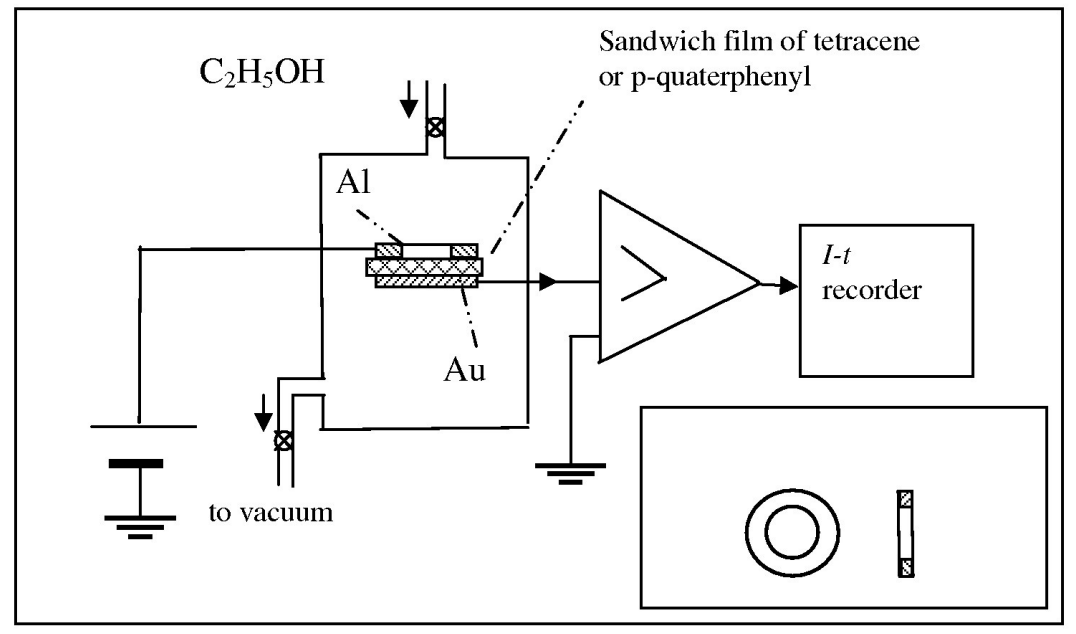

Fig. 2 Experimental set-up for activating the films and for recording the $I-t$ characteristics of the dark current induced by the activation process. Insert shows the construction of the upper aluminium electrode.

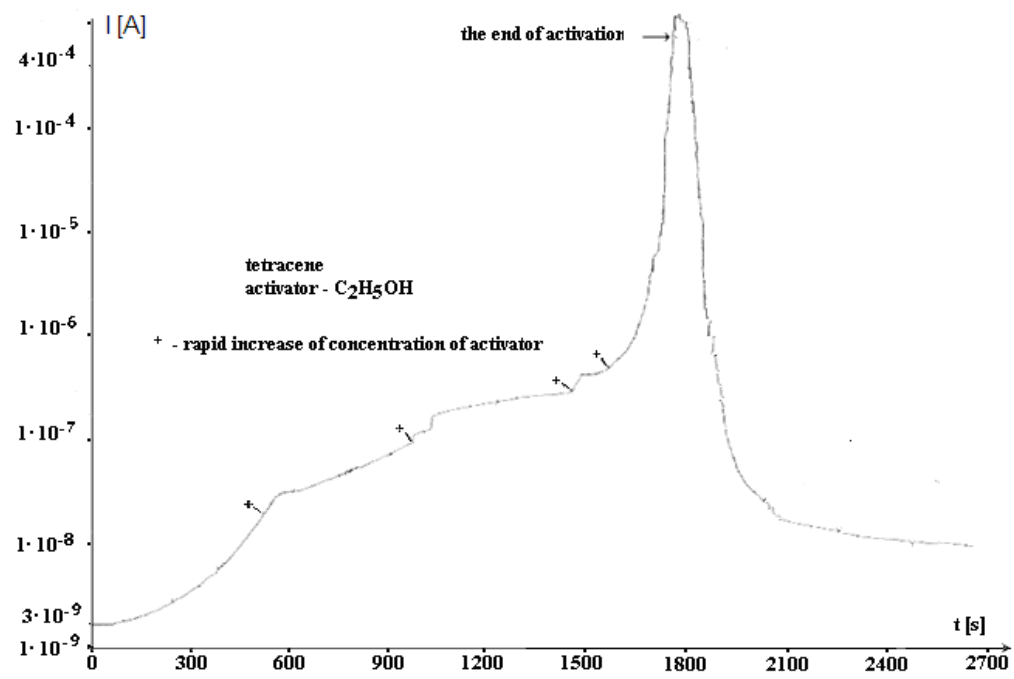

Fig. 3 Kinetics of the dark current passing through the tetracene film in the course of the experiment.

activation caused by chemisorption of oxygen or steam. A similar, but considerably stronger effect was observed in other works [10-12] for the activation of the same kinds of layer with ethanol. In our study we observed a rapid increase of the current after each step of pressurization under the influence of ethanol (Figs. 3, 4).

At all the steps saturation of the current was observed within very short time $(<1 \mathrm{~min})$. It means that dynamic equilibrium was obtained between the layer and the alcohol vapour. But the value of the current was equilibrated to the higher level for every new portion of $\mathrm{C}_{2} \mathrm{H}_{5} \mathrm{OH}$ let into the chamber. Therefore we can consider that the level at which equilibrium is achieved depends on the concentration of ethanol. The observed time dependence of the current may indicate injection of carriers due to the adsorption of a new portion of ethanol and subsequent diffusion of the carriers inside the layer. If the process had been connected only with physisorption, then the increase of the current would not have been so rapid because of the low energy of this process, typically below $0.1 \mathrm{eV}$. If the process was connected with strong chemisorption, then we would not observe rapid increase of the current after each step of pressurization, since a permanent monolayer of chemisorbed $\mathrm{C}_{2} \mathrm{H}_{5} \mathrm{OH}$ would prevent the next delivery of carriers to the layer. For very thin aromatic layers a decrease of the conductivity was observed for cyclic organic molecules under the influence of ethanol at different pressures [7]. So we can assume that strongly adsorbed ethanol makes layers not conducting. There are three main possibilities for the adsorption mechanism between the adsorbate (ethanol) and the adsorbent acene: dipole-induced dipole interaction, non polar van der Waals attraction, and hydrogen 


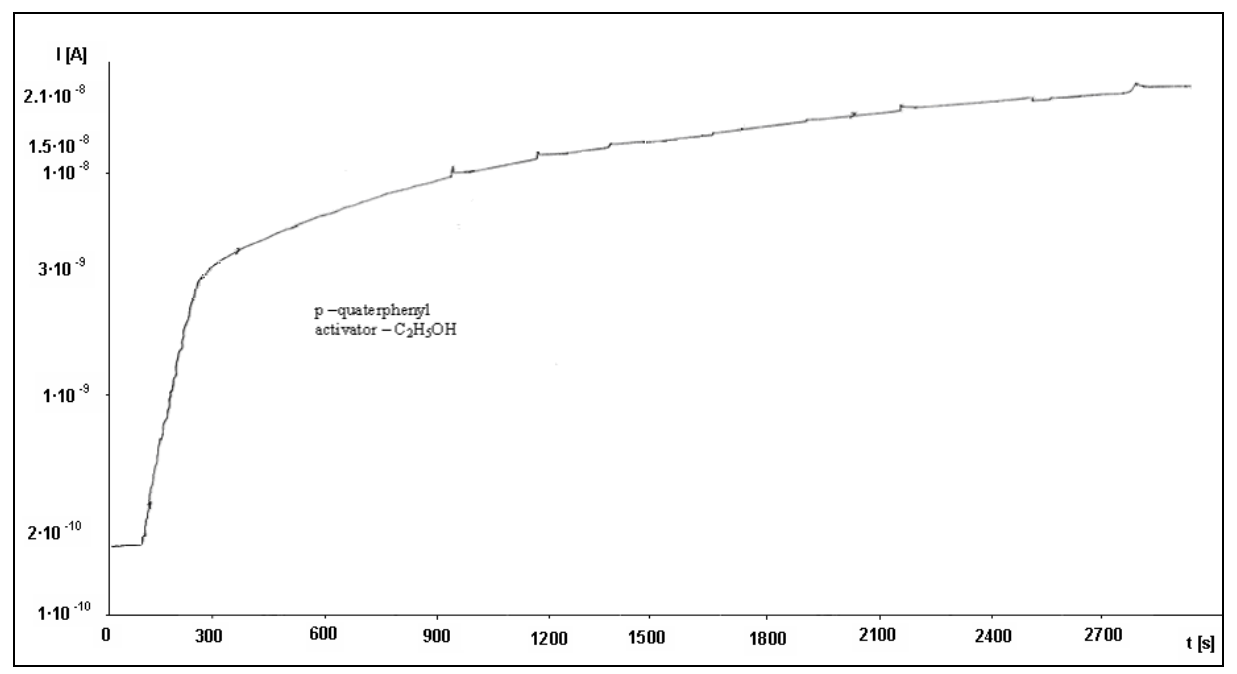

Fig. 4 Kinetics of the dark current passing through the p-quaterphenyl film in the course of the experiment.

bonding. Hydrogen bonding is more probable, occurring between the oxygen of the hydroxyl group of the alcohol and a whole single ring of the adsorbent molecule [8]. Such kind of bonding has a binding energy in the order of $0.1-0.5 \mathrm{eV}$, and is connected with concentrating the electron charge in the space near the hydrogen atom $[8,13]$. If the next molecule of ethanol collides with an adsorbed molecule of ethanol, then the centre of positive charge will rapidly move in the direction opposite to the layer, and the new mechanical system of two or more alcohol molecules can convert dynamic energy into potential energy. The Coulomb interaction of such a dimolecular (or more sophisticated) system with an acene layer will diminish and enable conversion from translational to internal energy, which will liberate the ionized dimolecular system from layer attraction, the fluctuation of the charge remaining on the surface. This process of injecting charge can be connected with equilibrium covering of the surface $\sigma$. If we assume that covering of the surface $\sigma$ is proportional to the time of adhesion of the molecule $\tau$, and to the molecular flux of vapour $f(\sigma=\tau \cdot f)$, then:

$\sigma=p \cdot\left[\mathrm{N}_{\mathrm{A}} /(2 \cdot \pi \cdot m \cdot \mathrm{k} \cdot T)\right]^{1 / 2} \cdot \tau_{0} \cdot \exp \left(\Delta H_{\mathrm{ads}} /(\mathrm{k} \cdot T)\right)$

where: $p$ - pressure, $\mathrm{N}_{\mathrm{A}}-$ Avogadro constant, $m-$ molecular weight, $\mathrm{k}-$ Boltzmann constant, $T$ temperature, $\Delta H_{\mathrm{ads}}$ - heat of adsorption for one adsorbate molecule, and $\tau_{0}=10^{-13} \mathrm{~s}$. With the assumption that the increase of the current over the initial value $I_{0}$ is proportional to the covering of the surface $\sigma$, the enthalpies of adsorption, determined from the slope of the $I-t$ curve for $\mathrm{C}_{2} \mathrm{H}_{5} \mathrm{OH}$ adsorption dependence (see Figs. 3, 4), are nearly identical for tetracene and for p-quaterphenyl: $84.4 \mathrm{~kJ} / \mathrm{mol}$ and $77.4 \mathrm{~kJ} / \mathrm{mol}$, respectively. The kinetics of the normalized current rise are nearly identical (see Fig. 5). This suggests that the number of adsorbed molecules is rather proportional to the number of rings or $\pi$ bonds of the adsorbing molecule than to the number of carbon atoms in the molecule.
The hypothetical transfer of charge to the layer described above can be supported based on the catastrophe theory founded by René Thom. New injections of charge from adsorbed alcohol molecules are possible due to the high asymmetry of the geometry of the process and are connected with transfer of the $\pi$ bonding electron taking part in the attraction of the ethanol molecule into the bulk of the film. Repulsive energy due to the Pauli exclusion principle would be a factor causing the activation of directional conductivity under the conditions of continuous delivery of charge to the layer. The topology of the system favours transfer of charge into the bulk. The mechanism described above may approve the assumption in the adiabatic approach that the adsorbing molecules do not interact with each other in the plane situated at the distance $r$ (in the range from 2 to $3 \AA$, typical for hydrogen bonding) from the adsorbing ring [8]. This situation may be understood from the fact that the hydrogen bonds should be centered at the centres of the rings of the acenes.

The Schrödinger equation for an isolated system consisting of adsorbate ethanol, a proton and $\pi$ electrons of the adsorbing ring is given by:

$H \Psi=W \Psi$,

where $W$ is the eigenvalue of energy of the system.

In the adiabatic approximation when the electron processes are much faster than the mechanical ones the Hamiltonian $H$ in the Schrödinger equation is given by:

$$
H \Psi=\left\{\frac{\hbar^{2}}{2} \sum_{j=0}^{K} \frac{1}{I} \frac{\partial^{2}}{\partial \phi^{2}}+\frac{\hbar^{2}}{2 m} \sum_{i=0}^{K^{\prime}} \Delta_{i}+V_{\mathrm{H}}+V_{\mathrm{e}}\right\} \Psi,
$$

where the first term concerns the kinetic energy of rotation [14] of the clustering alcohol molecules ( $\hbar$ is the Planck constant, $I$ the reduced moment of inertia, $\phi$ the torsion angle). The second term is connected with translational energy and vibration 


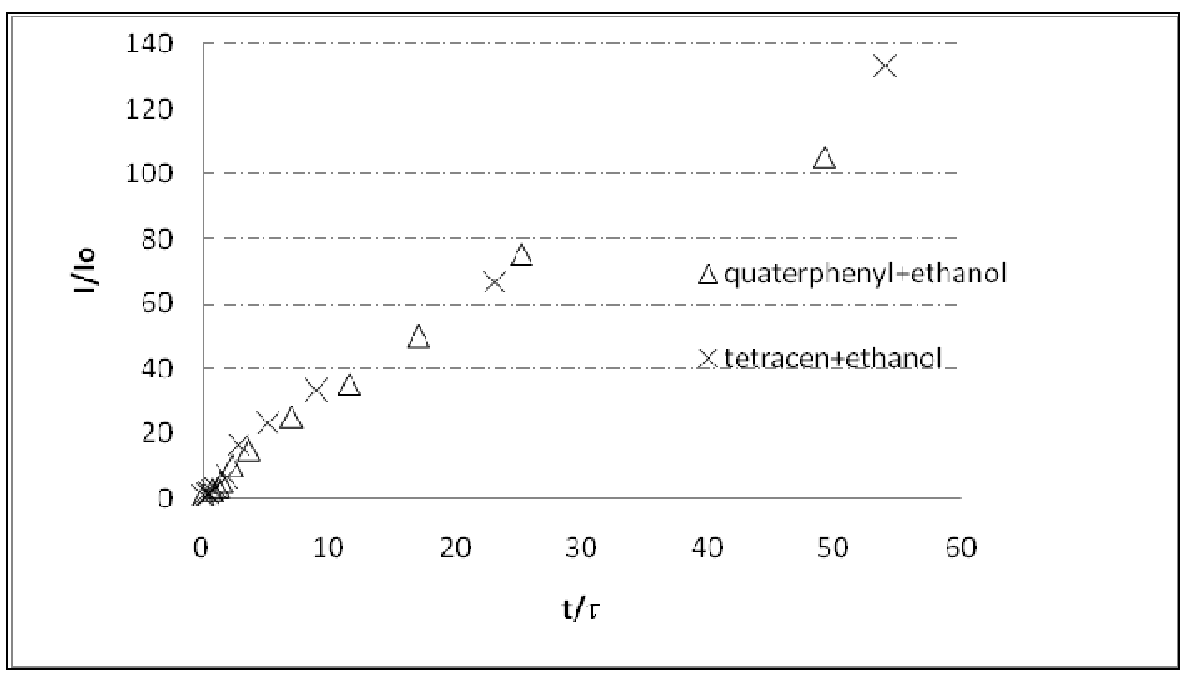

Fig. 5 Normalized dark current flow through a layer of ethanol-activated tetracene or p-quaterphenyl as a function of normalized activation time.

$\left(\Delta \equiv \partial^{2} / \partial x^{2}+\partial^{2} / \partial y^{2}+\partial^{2} / \partial z^{2}\right.$, where $x, y$ are axes parallel to the surface of the ring, and $z$ is normal to it). The next term, $V_{\mathrm{H}}$, defines the attracting interaction between the proton and ethanol, and the last term specifies the interaction between the cloud of electrons (located between the proton and the ring), the proton and ethanol. The second term is sensitive to the number $K^{\prime}$ of clustering molecules, because conversion of mechanical energy from the impinging molecule to internal energy of the cluster, which behaves like a statistical ensemble with the corresponding degree of freedom, is possible [15]. For hydrogen bonding the typical distance between conjugated molecules is in the range $2-3 \AA$ and the electrostatic Coulomb attracting term is much larger than the quantum exchange term and the minor induction and dispersive (van der Waals) terms. The electrostatic term is non-directional and enables bonding between hydrogen and several neighbouring atoms building a ring of the molecule (this is the case when hydrogen bonding connects the whole acene ring with the adsorbate molecule). $\AA$

The activation process is not fully reversible with the occurrence of the small "memory" effect so that we can consider the influence of a chemisorption process [1,11]. Based on the one order greater magnitude of the residual dark current after desorption, it was concluded that the nature of the surface interactions between the adsorbent and adsorbate molecules are rather complex. A detailed consideration of the data, however, led to the conclusion that the model of the adsorption process is consistent with the idea that two adsorption processes occur simultaneously, one with small activation energy and short lifetime, probably with hydrogen bonding, and the other one with larger activation energy, similar to adsorption of $\mathrm{O}_{2}$ or $\mathrm{H}_{2} \mathrm{O}$ steam $[1,2]$.
The dependence of the normalized dark current flow through a layer as a function of the normalized activation time (Fig. 5) shows identical behaviour for the two studied substances. This suggests similar mechanisms for the influence of adsorption on the electron structure of the adsorbing molecule, and similar numbers of active sites.

For larger concentrations of $\mathrm{C}_{2} \mathrm{H}_{5} \mathrm{OH}$ molecules and sufficiently long times (above $240 \mathrm{~s}$ ), an increase of the level of the current to $10^{-5} \mathrm{~A}$ was observed, i.e. an increase by approximately a factor 5, with a tendency to short-circuit in the film for higher values. This indicates that conducting channels had appeared, leading to durable damage of the layer.

If we do not bring the system to the condition of damage (in the electric sense hazards), then the activation of the layer should be considered as a reversible process with the occurrence of the "memory" effect (i.e. the output level of the intensity of the current for the same voltage was one order higher than it was before pressurisation). However, this effect disappears after repeated activation of the layer and no memory effect appears.

Similar phenomena have been observed for other aromatic hydrocarbons of low molecular weight.

The current flow $J$ through a thin dielectric layer of thickness $L$, induced by an applied voltage $V$, can be described by the known formula for non-trapping dielectrics:

$J=\frac{9}{8} \varepsilon \varepsilon_{0} \mu \frac{V^{2}}{L^{3}}$,

where: $\mu$ - the mobility of the charge carriers, $\varepsilon-$ the dielectric constant of the sample.

If only shallow traps occur in the studied dielectric the formula becomes:

$J=\frac{9}{8} \varepsilon \varepsilon_{0} \mu \vartheta \frac{V^{2}}{L^{3}}$, 
where $\vartheta(T)=n / n_{\mathrm{t}}, n-$ the total density of free charge carriers, $n_{\mathrm{t}}$ - the density of the trapped carriers, obviously within the limits of the foundation of the bound model of transport with the shallow traps localized in the forbidden gap.

For a more complicated expansion of states in the forbidden gap, the increase of the current has a more sophisticated representation and, for example, for an exponential expansion between the levels of energy $E_{i}$ and $E_{l}$, decreasing with increasing energy [12]:

$$
\rho(E)=\rho_{0} \exp \left(-\frac{E_{l}-E_{i}}{\mathrm{k} T_{\mathrm{c}}}\right),
$$

the formula should be

$$
\left.J=\frac{N_{\mathrm{c}} \mu \mathrm{e}}{L}\left(\frac{b}{N_{t}}\right) V^{\left(\frac{T_{\mathrm{c}}}{T}+1\right.}\right)\left[\exp \left(-\frac{E_{l}}{\mathrm{k} T}\right)\right],
$$

where: $b=\varepsilon \varepsilon_{0} \alpha \beta / \mathrm{e} L^{3}, 1<\alpha<2,1 / 2<\beta<1, N_{\mathrm{t}}-$ the number of localized states per volume unit, and $N_{\mathrm{c}}-$ the number of states in the band, e - the electron charge $T_{\mathrm{c}}-$ the characteristic temperature. If we activate the layer the density of localized states should obviously increase.

This is an approximation and the expression (within the scope of the above mentioned model) describing the value of the current flowing through the sample would have to be more complex.

The adsorption model predicts injection of carriers. The variation of the number of carriers would shift the Fermi level inside the layer and the mobility would increase. Such a mechanism is possible under the condition that hopping transport is the dominant mechanism of conductivity.

Verification of this hypothesis should be possible by measuring the mobility directly during the process of activation of the layer. Unfortunately such measurements could not be realised technically. It was only possible to measure the drift mobility for the p-quaterphenyl layers in a vacuum of $10^{-5}$ Torr and in air with a humidity of approx. $60-70 \%$, i.e. in the presence of polar molecules of aqueous steam $\left(\mathrm{H}_{2} \mathrm{O}\right)$ and oxygen molecules $\left(\mathrm{O}_{2}\right)$. For the layers of p-quaterphenyl in the arrangement of sandwich electrodes the Hall mobility was measured using the set-up presented in Fig. 2.

The mobility of electrons and holes in the layers was determined from the position of the kink in the obtained impulse. For both layers the drift mobility for holes in vacuum was obtained. For p-quaterphenyl, $\mu=2.0 \times 10^{-5} \mathrm{~cm}^{2} / \mathrm{Vs}$ with an activation energy of $0.04 \mathrm{eV}$, and in atmosphere the drift mobility $\mu=9.0 \times 10^{-5} \mathrm{~cm}^{2} / \mathrm{Vs}$; for tetracene in vacuum: $\mu=4 \times 10^{-4} \mathrm{~cm}^{2} / \mathrm{Vs}$ with an activation energy of $0.03 \mathrm{eV}$, and in atmosphere the drift mobility $\mu=7 \times 10^{-4} \mathrm{~cm}^{2} / \mathrm{Vs}$. The low value of the drift mobility and its low activation energy suggests that hopping charge transport takes place in a narrow band of localized states. According to the Mott-Davies model describing hopping transport in a narrow band of states at the Fermi level the drift mobility is given by: $\mu=\frac{1}{6} \frac{\mathrm{e} R^{2}}{\mathrm{k} T} \cdot v_{\mathrm{ph}} \cdot \exp (-2 \alpha \cdot R) \cdot \exp \left(-\frac{W}{\mathrm{k} T}\right)$, where $R$ is the average distance between the localized states, $\alpha$ is the decay constant of the localized state wave function, $W$ is the effective width of the band of localized states, $T$ is the temperature. Using the above equation and the geometrical formula $N\left(E_{\mathrm{F}}\right)=(3 / 4)\left(1 / R^{3} \cdot W\right)$ one can estimate the average distance between the localized states $R$ and the density of localized states at the Fermi level $N\left(E_{\mathrm{F}}\right)$. The values of $N\left(E_{\mathrm{F}}\right)$ obtained for the measurements in vacuum are close to $3 \times 10^{19} \mathrm{~cm}^{-3} \mathrm{eV}^{-1}$ for both of the studied acenes, while for the measurements carried out in air the values turned out to be nearly one order of magnitude higher. These values of the density of states are typical for many non-crystalline materials and seem to confirm the consistency of the model.

The fact that the mobility of the electrons in tetracene and holes in p-quaterphenyl increases can be interpreted as an increase of the density of localized states. This is in agreement with our earlier suggestion that the increase of conductivity resulting from the activation process may be the consequence of an increase of the number of localized states, which is of course possible if these are the states through the transport of carriers occurs [16].

The mean free path of an electron in anthracene is estimated to be of the same order of magnitude as the intermolecular spacing in tetracene and p-quaterphenyl, so when we observe an increase of the conductivity with increasing number of states it may not be realistic to describe charge carrier motion in terms of the band model. Therefore the obtained results may suggest hopping transport through localized states as the dominant kind of transport. A variation of the conductivity was observed due to adsorption of ethanol.

\section{Acknowledgements}

Great thanks to Prof. M. Wieczorek for support in the structural examinations and to Prof. J. Swiatek for valuable discussions.

\section{References}

[1] S. Kania, J. Kondrasiuk, G.W. Bąk, Eur. Phys. J. E 15 (2004) 439-442.

[2] S. Kania, J. Kondrasiuk, G.W. Bąk, Mol. Phys. Rep. 25 (1999) 93-98.

[3] J.J. Velasco-Velez, U. Kunze, T. Haas, T. Doll, Phys. Status Solidi A 207 (2010) 924-929.

[4] S. Maldonado, E. Garcia-Berrioz, M.D. Woodka, B.C. Brunschwig, N.S. Lewis, Sens. Actuators B 134 (2008) 521-531. 
[5] C.C. Cho, Chinese J. Phys. 33 (1995) 91-106.

[6] K.W. Kolasinsky, I. Harrison, A.V. Gavrilenko, C.E. Bonner Jr., V.I. Gavrilenko, Proc. SPIE 6320 (2006) 63200Q.

[7] H. Graaf, D. Schlettwein, N.I. Jaeger, Phys. Chem. Chem. Phys. 1 (1999) 1801-1806.

[8] M. Lewitt, M.F. Perutz, J. Mol. Biol. 201 (1988) 751-754.

[9] O. Bludsky, M. Rubes, Phys. Rev. Lett. B 77 (2008) 092103.

[10] S. Kania, Sci. Bull. Tech. Univ. Łódź 22 (2002) 31-37.

[11] S. Kania, J. Kuliński, Sci. Bull. Tech. Univ. Łódź 30 (2009) 65-72.
[12] S. Kania, J. Kuliński, Sci. Bull. Tech. Univ. Łódź 29 (2008) 45-56.

[13] B.G. Oliveira, M.L.A.A. Vasconcellos, R.R. Olinda, E.B.A. Filho, Struct. Chem. 20 (2009) 81-90.

[14] J. Li, A. Kazakov, F.L. Dryer, J. Phys. Chem. A 108 (2004) 7671-7680.

[15] C. Mair, J. Fedor, M. Lezius, P. Scheier, M. Probst, Z. Herman, T.D. Märk, New J. Phys. 5 (2003) 9.1-9.18.

[16] S. Kania, Visn. Lviv. Univ., Ser. Fiz. 40 (2007) 322-336.

Proceeding of the XVI International Seminar on Physics and Chemistry of Solids,

Lviv, June 6-9, 2010. 